\title{
A case of stage 4 gastric carcinoma (adenosquamous type) - rare clinical entity
}

\author{
S. Jaya Kiruthiga ${ }^{1}$, R. Rajkamal ${ }^{2}$
}

${ }^{1}$ PG Student, ${ }^{2}$ Assistant Professor, Dept. of Community Medicine, Sree Balaji Medical College \& Hospital, Chennai, Tamil Nadu

\author{
*Corresponding Author:
}

Email: jaikeerthi07@gmail.com

\begin{abstract}
Globally gastric carcinoma is the fifth leading cause of cancer and the third leading cause of death from cancer making up $7 \%$ of cases and 9\% of deaths. In 2012 it occurred in 950,000 people and caused 723,000 deaths. Before the 1930s in much of the world, it was the most common cause of death from cancer. Rates of death have been decreasing in many areas of the world. In this, we are going to discuss about a case of $70 \mathrm{yr}$ old woman presenting with mass in the epigastrium was diagnosed to be stage 4 adenosquamous type of gastric carcinoma who has undergone subtotal gastrectomy with chemotherapy and radiotherapy.
\end{abstract}

Keywords: Gastric carcinoma, Gastrectomy

\section{Introduction}

Most gastric tumors are adenocarcinomas. Primary gastric adenosquamous carcinoma is a rare malignancy. It constitutes less than one percent of all gastric carcinomas and its clinical presentation is the same as adenocarcinom. Stage IV stomach cancer has spread to distant organs, a cure is usually not possible. But treatment can often help keep the cancer under control and help relieve symptoms. This include surgery, such as a gastric bypass or even a subtotal gastrectomy. Chemo and/or radiation therapy can often help shrink the cancer and relieve some symptoms as well as help patients live longer, but is usually not expected to cure the cancer. Combinations of chemo drugs are most commonly used. Targeted therapy can also be helpful in treating advanced cancers. Even if treatments do not cure the cancer, there are ways to relieve pain and symptoms from the disease. Patients should inform about their symptoms to the doctor, so they can be treated accordingly. Nutrition is another concern for many patients with stomach cancer. nutritional counseling of placement of a tube into the small intestine to help provide nutrition for those who have trouble eating, if needed.

\section{Case Report}

A case of $70 \mathrm{yr}$ old woman presenting with mass in the epigastrium moving with respiration associated with nausea, vomiting, weight loss for two months duration. $\mathrm{He}$ also complaints of dysphagia, anorexia, early satiety, altered bowel habits, maelena, bleeding per rectum. On general examination pallor(+), lymph node enlarged mainly in left supraclavicular $(+)$ (Virchow node), cervical node(+). On per abdomen examination: a mass in epigastrium moves with respiration which is hard, non-tender, irregular in shape, liver enlarged in size, ascites (+), upper GI endoscopy revealed ulcerative lesion. CT scan showed direct invasion to the pancreatic body, lymph node metastasis mainly in the gastroesophageal junction. Biopsy specimen showed adenosquamous type of gastric carcinoma. Subtotal gastrectomy done which was not curative and he underwent chemotherapy and radiotherapy.

Upper GI Endoscopy: Ulcerative Lesion (Stage 4 Carcinoma)

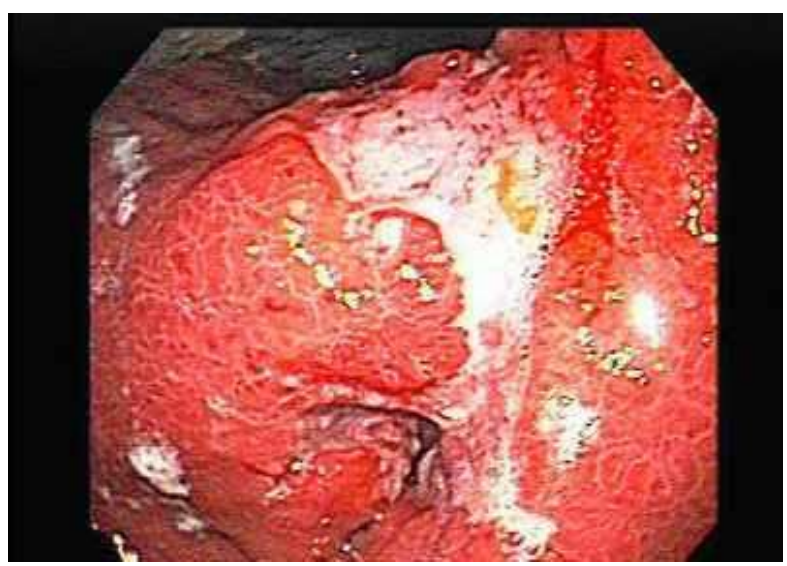

\section{Histopathology}

Glandular and squamous component - adenosquamous carcinoma

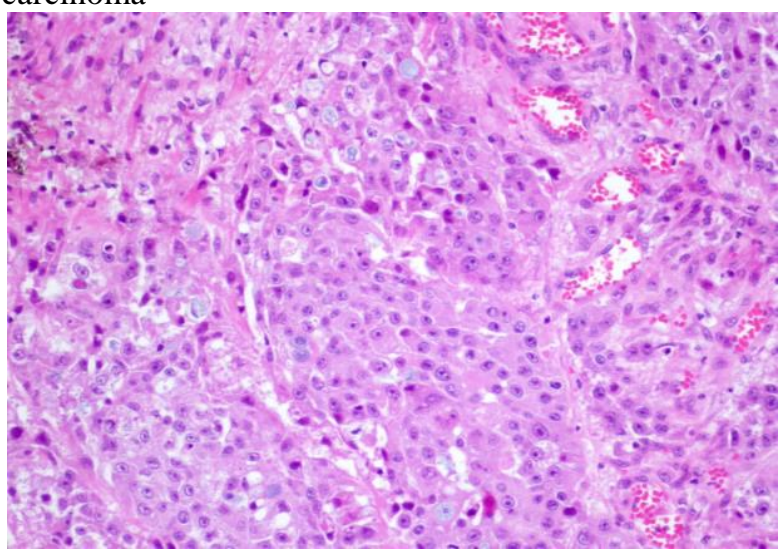




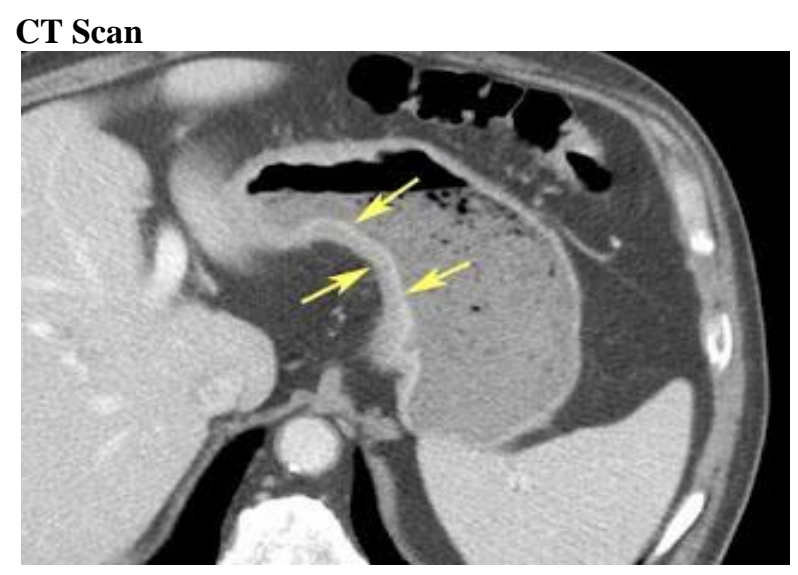

CT Scan: Showing Stage 4 Gastric Carcinoma

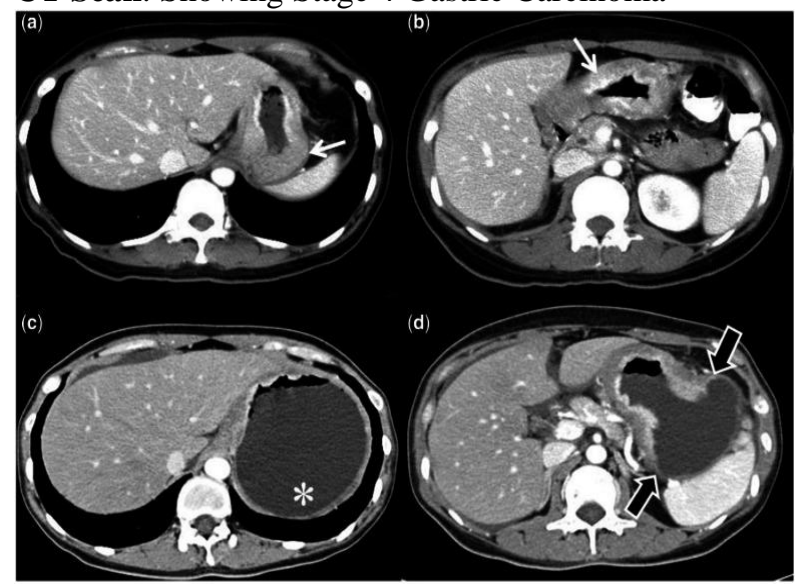

\section{Discussion}

We have reported a patient with advanced stage 4 gastric adenosquamous type of carcinoma which is a rare one. For the diagnosis of a true adenosquamous carcinoma, it is necessary to confirm the presence of a mixed-pattern carcinoma (glandular and squamous component), without esophageal involvement and without adenosquamous carcinoma in other organs. Besides this, it is also necessary for the squamous component to be present in over 25 percent of the tumor mass. We report the case of a 70-year-old woman with an adenosquamous carcinoma extending to her serosa, with nodal and hepatic metastasis, and also with lymphatic and venous invasion. The adenosquamous carcinoma usually behaves like an aggressive adenocarcinoma and presents with early lymphatic metastization. As such, the disease was considered metastized and the neoplasia classified as adenosquamous gastric carcinoma, pT3N1M1 (stage IV), which suggests a survival rate below seven percent at five years.

\section{Conclusion}

We report the case of a woman with a primary adenosquamous carcinoma of the stomach; rare in women and in the gastric antrum, and unique in its metastasis pattern, which was predominantly squamous.
The hypothesis that a particular histological type of gastric cancer might arise from stem cells could become a possible field of research in the oncological disease of the stomach. Patients with risk factors for gastric cancer and problems with epigastric pain, unintentional weight loss, or other suspicious symptoms should undergo diagnostic work-up. Endoscopy with biopsy mapping should be considered to look for multifocal gastric metaplasia in patients who are asymptomatic but at high risk of developing gastric carcinoma because of a positive family history, racial or ethnic origin for gastric cancer.

\section{References}

1. Namatame $K$, Ookubo $M$, Suzuki $K$, Sagawa $H$, Nagashima T, Kataba Y, Maruyama U, Watanabe H: A clinicopathological study of five cases of adenosquamous carcinoma of the stomach. Gan No Rinsho. 1986,32:170175.PubMedGoogle Scholar

2. Nishiwaki $\mathrm{H}$, Iriyama $\mathrm{K}$, Mori $\mathrm{H}$, Teranishi $\mathrm{T}$, Yano $\mathrm{H}$, Suzuki H: Adenosquamous carcinoma of the stomach. Gan No Rinsho. 1985,31:1802-1804.PubMedGoogle Scholar

3. Toyota N, Minagi S, Takeuchi T, Sadamitsu N: Adenosquamous carcinoma of the stomach associated with separate early gastric cancer (type IIc). J Gastroenterol. 10.1007/BF01211195.View ArticlePubMedGoogle Scholar

4. Mori M, Iwashita A, Enjoji M: Squamous cell carcinoma of the stomach: report of three cases. Am J Gastroenterol. 1986,81:339-342.PubMedGoogle Scholar

5. Straus R, Heschel S, Fortmann DJ: Primary adenosquamous carcinoma of the stomach. A case report and review. Cancer. 1969, 24: 985-995. 10.1002/10970142(196911)24:5<985::AID-

CNCR2820240518>3.0.CO;2-4.

6. Faria GR, Eloy C, Preto JR, Costa EL, Almeida T, Barbosa J, Paiva ME, Sousa-Rodrigues J, Pimenta A. Primary gastric adenosquamous carcinoma in a Caucasian woman: a case report. Journal of medical case reports. 2010 Oct 29;4(1):351. 